\title{
A Method for Analyzing Code Homology in Genealogy of Evolving Software
}

\author{
Masatomo Hashimoto ${ }^{1}$ and Akira Mori $^{2}$ \\ 1 AIST Tokyo Waterfront, 2-3-26 Aomi, Koto-ku, Tokyo 135-0064, Japan \\ m.hashimoto@aist.go.jp \\ 2 AIST Tokyo Akihabara Site, 1-18-13 Sotokanda, Chiyoda-ku, \\ Tokyo 101-0021, Japan \\ a-mori@aist.go.jp
}

\begin{abstract}
A software project often contains a large amount of "homologous code", i.e., similar code fragments distributed in different versions or "species" sharing common ancestry. Code homology typically arises when the code is inherited, duplicated, and patched. In this paper, we propose an automated method for detecting and tracking homologous code in genealogy of evolving software using fine-grained tree differencing on source code. Such a tool would help software developers/ maintainers to better understand the source code and to detect/prevent inconsistent modifications that may lead to latent errors. The results of experiments on several large-scale software projects are reported to show the capability of the method, including BIND9 DNS servers, a couple of Java software systems jEdit and Ant, and the entire Linux device driver subsystem.
\end{abstract}

\section{Introduction}

A large software system often contains a large number of similar code fragments across many versions or branches. They are typically introduced when the code is inherited from previous versions, duplicated for programming convenience, and patched to correct common defects. We call such correspondence in the code descended from a common ancestry homology of code, by analogy with biology 1 .

Homologous code fragments or homologues may evolve in uniform or divergent manner as the development proceeds. If the evolution is uniform, it is likely that there exists a common programming logic and extra maintenance efforts are necessary 2 since further changes must be replicated on each code fragment to keep the system consistent. Even when the evolution is divergent, common characters of the code remain in later developments 3 .

Locating homologous code and tracking their course of change would help software developers/maintainers to better understand the source code and to detect/prevent inconsistent modifications that may lead to latent errors. The task can be difficult even when a versioning system such as CVS is in place to record change descriptions since such information is too coarse to compare and

D.S. Rosenblum and G. Taentzer (Eds.): FASE 2010, LNCS 6013, pp. 91-106, 2010.

(C) Springer-Verlag Berlin Heidelberg 2010 
usually not associated with reference to concrete source code entities such as functions and methods [2].

The analysis of code clones is a well-researched topic related to code homology. Many algorithms and tools have been proposed for detecting code clones [4]. However, those methods are not well-suited for analyzing how clone regions evolve over time since maintaining clone relations is difficult when regions go through different modifications and do not remain the same. Clone detection must be performed on each version and discovered clone regions must be tracked in the later versions. To distinguish newly introduced clone regions from those lasting from previous versions involves an awkward task of adjusting similarity thresholds by heuristics 3. The method for tracking cloned code is relatively less explored with several exceptions 5 3678 despite its practical importance.

In this paper, we propose an automated method for detecting and tracking homologues in genealogy of evolving software using a fine-grained tree differencing tool called Diff/TS 9] for source code. Tree differencing is used for calculating edit sequences on abstract syntax trees that transform one revision into another. It also computes node mappings up to relabeling for tracking source code entities across revisions. The method allows us to identify inconsistent changes on homologues by comparing semantic change histories reconstructed from raw edit sequences.

By following ideas from biology, we classify homology into three categories: orthology, paralogy, and xenology. Orthology describes homology arising from branching activity, xenology from exchange of code across differ-

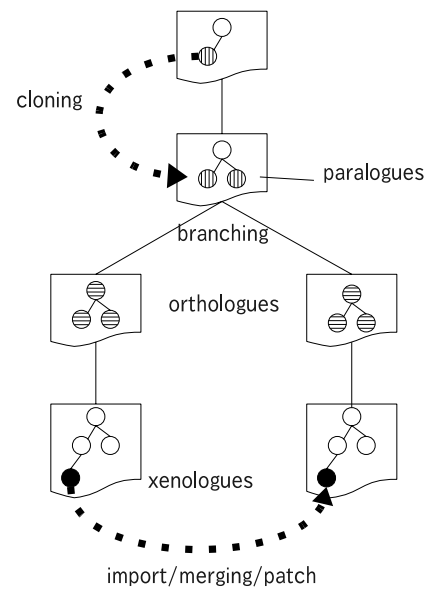

Fig. 1. Types of Code Homology ent branches, and paralogy from duplication in a single branch. See Fig. 1 for illustration.

According to the classification, we implemented procedures for analyzing homologues. To show the capability of the method, the results of experiments on several large-scale software are reported including BIND9 DNS servers, a couple of Java software systems jEdit and Ant, and Linux device drivers. Xenology is investigated with BIND9 and paralogy is investigated with jEdit, Ant, and Linux device drivers.

The results shows that the proposed method is efficient enough to analyze the entire device driver subsystems in 32 versions of Linux from 2.6.0 to 2.6.31, each of which consists of millions of lines of source code. Several inconsistencies in Linux serial drivers have been detected that violate a crucial development policy change concerning kernel locking. It is also shown that the method produces better analysis results compared to existing code clone trackers. In fact, 
the system could track not only all clone regions reported in the previous literature [563178], but also regions that escaped from previous analysis 6].

To summarize, the contributions of the paper are:

- proposal of the notion of code homology to categorize similar code in genealogy of evolving software,

- development of an automated method for detecting and tracking homologues, and

- development of an automated method for reconstructing and comparing finegrained change histories for homologues.

The rest of the paper is organized as follows. Prerequisites for tree differencing used in code homology analysis are explained in Sect. 2. Section 3 describes the method for code homology analysis. The results of the experiments is reported in Sect. 4. After related work is reviewed in Sect. 5. we conclude in Sect. 6.

\section{Tree Differencing}

We regard a version of a software system as a set of abstract syntax trees (ASTs) which correspond to source files (compilation units) and also as a directory tree which consists of source files. Tree differencing plays an important role in code homology analysis, which consists of three steps: detecting homologous code, tracking homologous code, and tracking changes of homologous code. We enumerate below the required functions in each step, whose details are explained in Sect. 3. Tree differencing is responsible for 1-(a), 1-(b), 2-(a), and 3-(a) in code homology analysis.

1. Homologous code detection

(a) discovering common structures between a pair of ASTs - for orthologues

(b) differencing a pair of ASTs - for xenologues

(c) detecting code clones - for paralogues

2. Code fragment tracking

(a) mapping nodes in one AST to corresponding nodes in another AST

(b) detecting cloning activities

(c) mapping line ranges in source code to the corresponding sub-ASTs and vice versa

3. Change history reconstruction

(a) deriving higher level descriptions from low level descriptions for changes between a pair of ASTs

A fundamental function of a tree differencing algorithm is to calculate a sequence of edit operations (called edit sequences) that transforms $T_{1}$ into $T_{2}$ for a given pair of trees $T_{1}$ and $T_{2}$. The basic edit operations include 1 ) relabeling, 2 ) deletion, and 3) insertion of nodes, and 4) moves of subtrees. An edit sequence between two ASTs may be regarded as difference or similarity between them. Tree differencing also computes a set of matched pairs of nodes between target trees. We call such a set of matched pairs of nodes a node map, which may be 
regarded as a (partial) finite mapping. Note that labels of a matched pair of nodes do not necessarily coincide. Node maps are constrained to preserve the structure of target trees up to relabeling. A common part of a pair of ASTs $T_{1}$ and $T_{2}$ with respect to a node map $M$ between them is defined as a pair of sets of nodes $(\operatorname{dom}(M), \operatorname{cod}(M))$, where $\operatorname{dom}(X)$ and $\operatorname{cod}(X)$ denote domain and codomain of mapping $X$, respectively.

We can use any tree differencing algorithms or tools that satisfy the requirements for code homology analysis described above. In this study, we used a tree differencing system called Diff/TS 9. Diff/TS approximates and extends an optimal tree comparison algorithm with heuristics driven control configurable for multiple programming languages. Diff/TS is capable of processing Python, Java, $\mathrm{C}$ and $\mathrm{C}++$ projects, and provides all required functions but $3-(\mathrm{a})$. In order to reconstruct higher level description of source code changes from low level edit sequences computed by tree differencing, we developed a module for classifying changes in $\mathrm{C}$ programs into approximately 80 change types following the ideas of Fluri and Gall [2]. Change types are defined according to edit operations and syntactical information embedded in ASTs. For example, a change type function call inserted is defined as insertion of a subtree corresponding to function call and return value changed as some edit operation(s) on AST node(s) in a subtree corresponding to a return value.

\section{Code Homology Analysis}

This section presents procedures for detecting and tracking homologous code in a given set of branches. An overview of our key techniques for code homology analysis is also provided.

The tree differencing algorithm allows us to distinguish between the preserved portion (up to relabeling) and the added/deleted portion between a pair of versions, and to compute edit operations which transform the one into another. We employ a "double-differencing" technique to identify xenologues. First, we pairwise compute preserved portions between relative versions and conclude that the preserved portion found closest to the most recent common ancestor represents orthologues. Then, we compute differences between preserved portions to identify added code fragments as xenologues. See Fig. 2 for illustration, where version $A$ branches into versions $B$ and $C$, which then evolve into $B 1$ and $C 1$, respectively. A vertical dashed line denotes difference, in which a black triangle represents code addition and a white triangle represents code deletion. A horizontal dashed line denotes common code segments, i.e., homologues between relative versions. Segments that have been newly added in the homologues suggest existence of merged or patched code, which we call xenologues (e.g., a shaded black triangle in Fig. 2). Other segments that have disappeared from the homologues suggest existence of individually modified code. In general, we cannot decide whether xenologues are originated from code exchange or simultaneous patch application without manually inspecting available documents such as change-logs or development histories. 


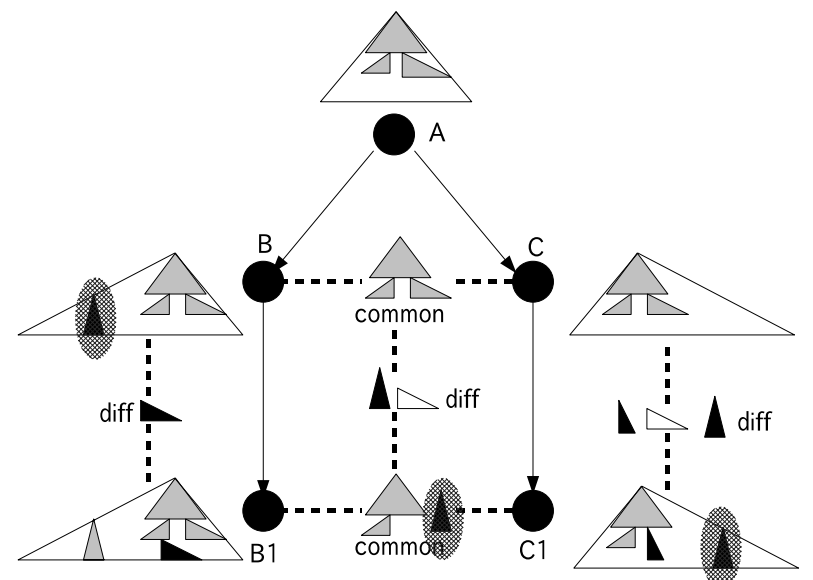

Fig. 2. Double-Differencing Between Branches

Paralogues, that is, code clones or duplicated code, are detected in a different manner. For the (given) initial version of the software, we apply existing code clone detection tools and accept the outputs as clone groups generated before the first version. For identifying cloning activities after the first version, we first compute added code fragments by tree differencing, and then find its potential duplication origins in the previous versions. A common token sequence matching algorithm is used for this.

We introduce several notations and terms needed for the rest of the paper. The set of nodes in $A$ is denoted by $\mathcal{N}(A)$. By $\mathcal{I}(A)$, we denote the set of post-order indexes of an $\operatorname{AST} A$. We identify nodes with their post-order indexes. Let $v$ be a version of software system. We denote the set of ASTs corresponding to source files contained in $v$ by $\mathcal{A}(v)$. For a tree $A$ and $S \subseteq \mathcal{N}(A),\left.A\right|_{S}$ denotes the tree obtained from $A$ by removing all nodes that do not belong to $S$. We introduce two wrapper functions of Diff/TS denoted by $\Delta$ for ASTs and $\Delta_{d}$ for directory trees. For ASTs $A_{1}$ and $A_{2}, \Delta\left(A_{1}, A_{2}\right)$ computes a triple $(M, D, I)$, where $M$ denotes a node map such that $M \subseteq \mathcal{I}\left(A_{1}\right) \times \mathcal{I}\left(A_{2}\right)$, $D$ a set of deleted components, and $I$ a set of inserted components. For versions $v_{1}$ and $v_{2}, \Delta_{d}\left(v_{1}, v_{2}\right)$ computes $(M, D, I)$, where $M$ denotes a node map such that $M \subseteq \mathcal{A}\left(v_{1}\right) \times \mathcal{A}\left(v_{2}\right), D$ a set of deleted source files, and $I$ a set of inserted source files. Note that internal nodes (directories) are omitted from $M$. We extend the domain of $\Delta$ to the set of versions: $\Delta\left(v_{1}, v_{2}\right)=\left\{\left(A_{1}, A_{2}, M, D, I\right) \mid(M, D, I)=\Delta\left(A_{1}, A_{2}\right)\right.$, where $\left.\left(A_{1}, A_{2}\right) \in M_{d},\left(M_{d}, D_{d}, I_{d}\right)=\Delta_{d}\left(v_{1}, v_{2}\right)\right\}$.

In order to detect homologues, we compute common code structures (CCSs) between versions. A CCS between two ASTs $A_{1}$ and $A_{2}$ with respect to a node map $M$, denoted by $\operatorname{CCS}_{M}\left(A_{1}, A_{2}\right)$, is defined as a pair of trees $\left(S_{1}, S_{2}\right)$ where $S_{1}=\left.A_{1}\right|_{\operatorname{dom}(M)}$ and $S_{2}=\left.A_{2}\right|_{\operatorname{cod}(M)}$. A CCS between two versions is defined as a set of CCSs between ASTs which corresponds to source files matched 


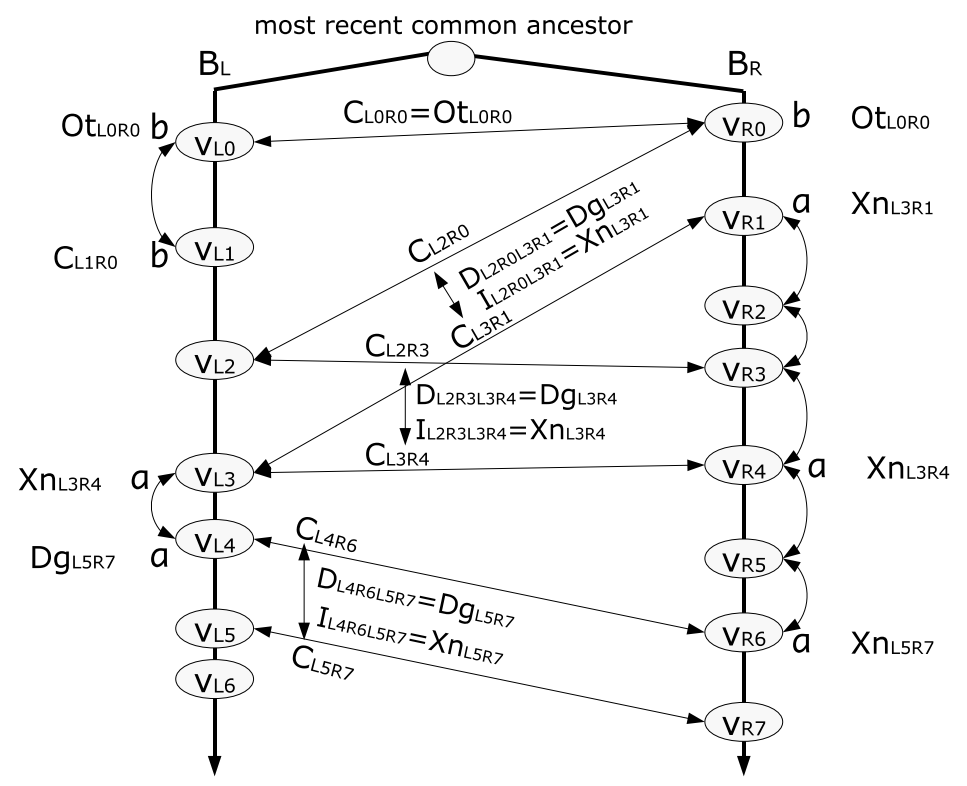

Fig. 3. Detecting Homologues

by $\Delta_{d}$. We can compute a CCS between versions $v_{1}$ and $v_{2}$ by first computing $\left(M_{d}, D_{d}, I_{d}\right)=\Delta_{d}\left(v_{1}, v_{2}\right)$, and then computing $(M, D, I)=\Delta\left(A_{1}, A_{2}\right)$ and $\left(\left.A_{1}\right|_{\operatorname{dom}(M)},\left.A_{2}\right|_{\operatorname{cod}(M)}\right)$, for each $\left(A_{1}, A_{2}\right) \in M_{d}$.

\subsection{Detecting Homologues}

An orthologue is defined between versions of different branches. For a pair of branches $B_{L}$ and $B_{R}$ diverged from a common ancestor, an orthologue between the oldest versions of $B_{L}$ and $B_{R}$ is defined as a CCS between the oldest versions. See Fig. 3, where $C_{L 0 R 0}$ and $\mathrm{Ot}_{L 0 R 0}$ denote a CCS and an orthologue between versions $v_{L 0}$ and $v_{R 0}$ of branches $B_{L}$ and $B_{R}$. Once we have obtained an orthologue between the oldest versions, orthologues between other versions of $B_{L}$ and $B_{R}$ is obtained by tracking the oldest orthologue. Our method of tracking code fragments is described in Sect. 3.2

In order to detect xenologues, we must perform differencing one more time on CCSs. Let $B_{L}$ and $B_{R}$ be branches that stem from a common ancestor (See Fig. (4). For versions $v_{L b}$ and $v_{R b}$, we compute $\operatorname{Xn}\left(v_{L b}, v_{R b}\right)$ which denotes the set of xenologues between $v_{L b}$ and $v_{R b}$. We let $\Delta_{d}\left(v_{L b}, v_{R b}\right)=\left(M_{L b R b}, D_{L b R b}, I_{L b R b}\right)$ where $M_{L b R b}=\{(1,1),(5,3)\}$, where nodes are indicated by indexes (by postorder traversal). Similarly, for $\left(v_{L a}, v_{R a}\right),\left(v_{L a}, v_{L b}\right)$, and $\left(v_{R a}, v_{R b}\right)$, we let $M_{L a R a}=\{(1,1),(3,3)\}, M_{L a L b}=\{(1,2),(3,5)\}$, and $M_{R a R b}=\{(3,3)\}$. Among the pairs contained in $M_{L b R b}$, only $(5,3)=\left(A_{L b 5}, A_{R b 3}\right)$ is able to form a commutative diagram consisting of dashed arrows in Fig. 4 . Similarly in $M_{L a R a}$, only $\left(A_{L a 3}, A_{R b 3}\right)$ form a diagram. We apply $\Delta$ to $\left(A_{L b 5}, A_{R b 3}\right)$ and $\left(A_{L a 3}, A_{R a 3}\right)$ to 


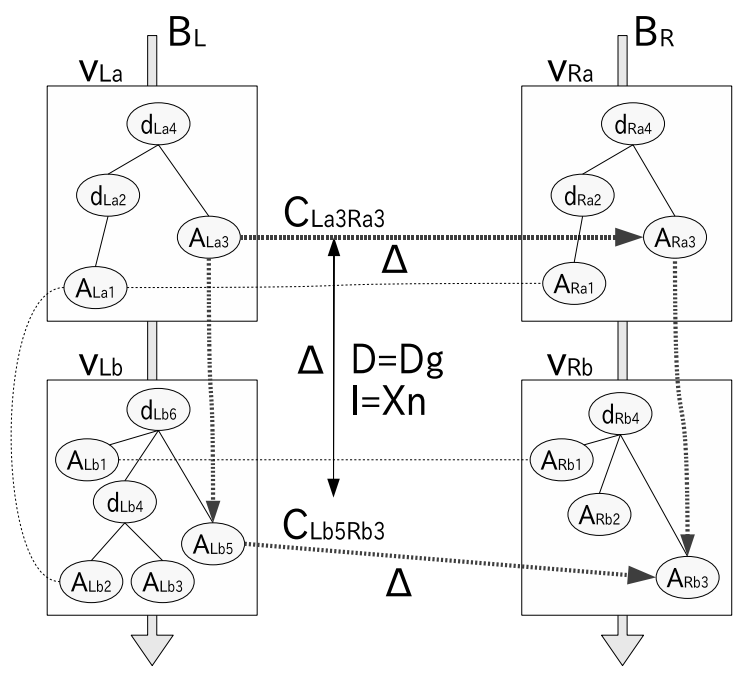

Fig. 4. Differencing Common Code Structures

obtain CCSs for them. Let $M_{b}=M_{L b 5 R b 3}$ and $M_{a}=M_{L a 3 R a 3}$ be node maps obtained from the applications, respectively. By definition, $\mathrm{CCS}_{M_{b}}\left(A_{L b 5}, A_{R b 3}\right)$ $=\left(\left.A_{L b 5}\right|_{\operatorname{dom}\left(M_{b}\right)},\left.A_{R b 3}\right|_{\operatorname{cod}\left(M_{b}\right)}\right)$ and similarly we have $\operatorname{CCS}_{M_{a}}\left(A_{L a 3}, A_{R a 3}\right)$ $=\left(\left.A_{L a 3}\right|_{\operatorname{dom}\left(M_{a}\right)},\left.A_{R a 3}\right|_{\operatorname{cod}\left(M_{a}\right)}\right)$. We let $C_{L b 5 R b 3}=\left.A_{L b 5}\right|_{\operatorname{dom}\left(M_{b}\right)}$ and $C_{L a 3 R a 3}=$ $\left.A_{L a 3}\right|_{\operatorname{dom}\left(M_{a}\right)}$. Finally, we apply $\Delta$ to $C_{L a 3 R b 3}$ and $C_{L b 5 R b 3}$. Let $(M, D, I)=$ $\Delta\left(C_{L a 3 R b 3}, C_{L b 5 R b 3}\right)$. I corresponds to $\mathrm{Xn}\left(A_{L b 5}, A_{R b 3}\right)$ and $D$ "degenerated" homologues between $A_{L b 5}$ and $A_{R b 3}$, denoted by $\operatorname{Dg}\left(A_{L b 5}, A_{R b 3}\right)$. Note that we can choose $\left.A_{R b 3}\right|_{\operatorname{cod}\left(M_{b}\right)}$ for $C_{L b 5 R b 3}$ or $\left.A_{R a 3}\right|_{\operatorname{cod}\left(M_{a}\right)}$ for $C_{L a 3 R a 3}$ since we ignore the difference of node labels in the node maps.

As mentioned in the beginning of Sect. 3, it is impossible to determine the origin of xenologues in general. For example, in Fig. 3. suppose that there exists some $a \in \mathrm{Xn}_{L 3 R 4}$ and it also exists $v_{L 3}$ through $v_{L 4}$, and $v_{R 1}$ through $v_{R 6}$. We can not decide whether $a$ is introduced by simultaneous patch application to $v_{L 3}$ and $v_{R 1}$ or by copying some part from a revision between $v_{R 1}$ and $v_{R 6}$ to $v_{L 3}$.

We use existing tools for identifying paralogues (code clones) in the initial version of the given software versions. For the versions descending from the initial versions, we use a code tracking method described in the next section for detecting cloning activities.

\subsection{Tracking Code Fragments}

Once the occurrence of a homologue is discovered, we look into a code continuum to inspect developments in the subsequent versions. A code continuum is a data structure created by composing differencing results across versions to record entire lifetime of a source code entity. A code continuum can be illustrated by a set of node continua, that is, threads representing the lifetime of AST nodes 


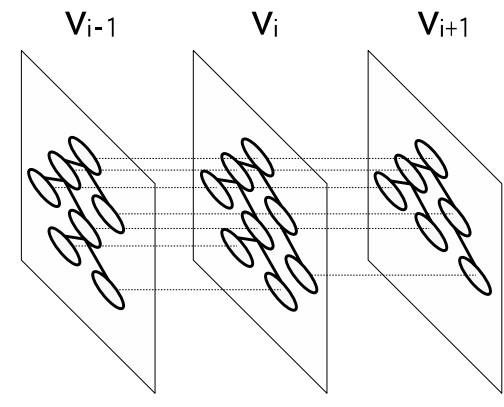

Fig. 5. Code Continuum
Alg. 1. Continuum Construction

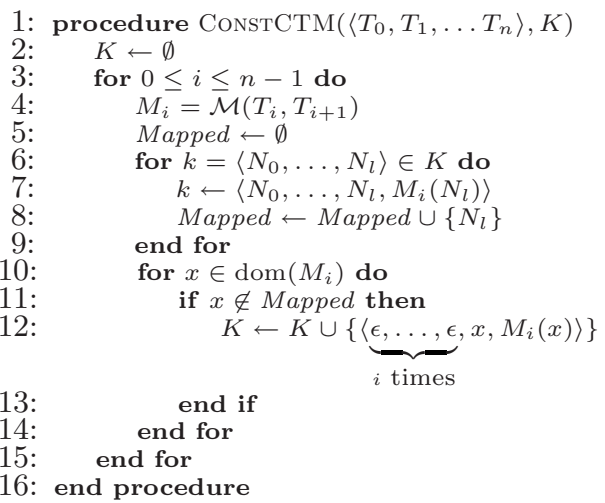

over time as in Fig. 5, where the beginning and the ending of a thread indicate the introduction and the removal of the AST node, respectively.

A node continuum is constructed for each AST node. For the same reason as we perform directory tree differencing, we construct a file continuum for each source code file. Each node/file continuum stores trace information of an AST node and a source file, respectively. A continuum for versions $v_{0}, \ldots, v_{n}$ is represented by a sequence of names $\left\langle N_{0}, \ldots, N_{n}\right\rangle$, where $N_{i}(0 \leq i \leq n)$ is a name of the node/file in version $v_{i}$. The node name is given by its index in post-order traversal and the file name by its path name. Non-existence of the node/file is represented by an empty name $\epsilon$ for convenience. An algorithm for constructing continua is shown in Alg. 1. In the description of continuum construction algorithm, $\mathcal{M}$ denotes a wrapper function of Diff/TS. For a pair of trees $T_{1}$ and $T_{2}, \mathcal{M}\left(T_{1}, T_{2}\right)$ computes a tree map between $T_{1}$ and $T_{2}$. The result is stored in $K$.

Since our AST nodes contain location information such as file names, line numbers, column positions and file offsets, continua make various analysis tasks easy including tracking corresponding source code entities on texts and reconstructing change sequences for a given code segment according to the results of source code

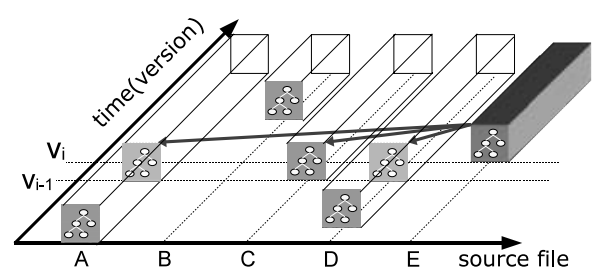

Fig. 6. Cloning Activity Detection tree differencing.

\subsection{Detecting Cloning Activities}

While traditional clone detection tools can discover code clones among given sets of software versions, they do not support tracking discovered code clones in the subsequent versions of software. To cope with the problem, we utilize 
code continua for identifying cloning activities by looking for the original code of cloning in the previous version by way of token based sequence matching.

Suppose that we analyze a sequence $v_{0}, v_{1}, v_{2}, \ldots, v_{n}$ of software versions. Clones that exist already in the initial version $v_{0}$ are marked using existing tools such as SimScant 1 for Java code and CCFinder [10] for C code. Cloning activities taking place between $v_{i-1}$ and $v_{i}(i \geq 1)$ are identified by the following procedure:

1. collect node continua starting from $v_{i}$ to form a tree $T$ included in the AST of $v_{i}$,

2. convert $T$ into a sequence $p$ of tokens by pre-order traversal,

3. compare $p$ with token sequences obtained from ASTs in $v_{i-1}$ by pre-order traversal using an $\mathrm{O}(\mathrm{ND})$ algorithm [11,

4. compute a score for each match by (number of matched tokens)/(number of tokens in $p$ ),

5. select the maximum score $s$ and if it exceeds the pre-defined threshold, concludes that $T$ is cloned from $v_{i-1}$.

The procedure is illustrated in Fig. 6. Note that code continua is collected to align pre-defined boundaries such as functions, methods, and classes to form subtrees.

\subsection{Constructing and Comparing Change Histories}

A change history for a code fragment is a sequence of change types obtained by accumulating change types whose locations are contained in the range of the fragment. In order to detect inconsistent changes, we define a similarity score between change histories as $2 \mathrm{~m} / t$, where $m$ is the number of matches calculated by a sequence matching algorithm and $t$ is the total number of change types in both histories. We regard a pair of code fragments as inconsistently modified if and only if either of the change histories is non-empty and the similarity score between the change histories is less than a specified similarity threshold.

\section{Experiments}

In this section, we present the results of code homology analysis on several open source software projects including BIND9 daemon (for xenologue detection), a couple of Java projects, jEdit2 and Ant (for paralogue detection and tracking), and the Linux kernel driver subsystem (for change history construction). The experiments are conducted in the following manner:

\footnotetext{
${ }^{1}$ http://www.blue-edge.bg/simscan/simscan_help_r1.htm

2 http://www.jedit.org/

3 http://ant.apache.org/
} 
Table 1. Sample Projects

\begin{tabular}{lccrrr}
\hline & lang. & \# of ver. & \multicolumn{1}{c}{ versions } & \# of src files & \multicolumn{1}{c}{ kSLOC } \\
\hline BIND9 & C & 26 & $9.0 .0-9.5 .0-P 2$ & $767-1,186$ & $153-260$ \\
jEdit & Java & 56 & $3.2 .2-4.3 .0$ pre17 & $279-532$ & $55-108$ \\
Ant & Java & 41 & $1.1-1.7 .1$ & $87-1,220$ & $9-125$ \\
Linux & $\mathrm{C}$ & 32 & $2.6 .0-2.6 .31$ & $12,424-23,752$ & $3,626-7,337$ \\
(drivers) & & & & $(3,285-8,116)$ & $(1,778-4,106)$ \\
\hline
\end{tabular}

1. Fill the local software repository with revisions of target software.

2. Xenologues are calculated by double-differencing (BIND9 only) and paralogues are calculated by backward code matching. Code clones detected in the first version are also treated as paralogues.

3. Paralogues are checked for inconsistent changes descending from the origin of the paralogues, which often account for latent bugs. Checking is done at the level of change type sequences described in Sect. 2 (except BIND9).

4. Discovered homologues and change histories go through human inspection for validation.

We used a PC with a pair of quad-core Intel Xeon CPU $(3.0 \mathrm{GHz})$ with $16 \mathrm{~GB}$ RAM running under Linux kernel 2.6.24 for these experiments.

The programming languages used for the software, the numbers and the ranges of analyzed versions, the numbers of source files, and kSLOC (ignoring comments and blank lines) of the initial and the latest versions of the target systems are shown in Table 1. For Linux, data for drivers subsystem are shown in parentheses.

\subsection{BIND9}

The main purpose of this experiment is to ascertain that our analysis can actually detect xenologues corresponding to merged or patched code fragments. ISC BIND (Berkeley Internet Name Domain) is an implementation of the Domain Name System (DNS) protocols. As of October, 2008, three release branches of BIND9, namely 9.2.x, 9.3.x, and 9.4.x are actively maintained. We selected 26 versions for analysis and our system detected 215 orthologues. We found that about $30 \%$ of them are degenerating, that is, decreasing in size. This indicates that $30 \%$ of commonly inherited code was modified in one or more branches and $70 \%$ of it is stable for generations. The system also detected 8,948 xenologues most of which $(98.27 \%)$ are relatively small in size $(<64$ nodes).

In the case of BIND9, it is likely that xenologues are introduced by patch applications such as security patches since multiple releases have been maintained in parallel. For example, a modification "Query id generation was cryptographically weak." (RT\#16915) is commonly included in CHANGES files contained in releases 9.2.8-P1, 9.3.4-P1, and 9.4.1-P1. As we expected, several xenologues in dispatch.c, out of 34 xenologues among 9.2.8-P1, 9.3.4-P1, and 9.4.1-P1, appear to be strongly related to that modification. 


\section{2 jEdit and Ant}

We collected 56 versions of jEdit from release 3.2.2 to 4.3pre17, and 41 versions of Ant from release 1.1 to 1.7.1. First, we compare the paralogue (code clone) tracking ability of our method with that of Duala-Ekoko and Robillard 6]. They implemented a system called CloneTracker which is capable of tracking code clones detected by clone detection tools. It identifies clone regions at the granularity of code blocks using heuristics based on the structural properties, lexical layout, and similarities of the clone region. They provided case studies of jEdit and Ant. A clone detection tool called SimScan was used to detect code clones in the initial versions. Then, they selected five clone groups, which were tracked across the subsequent versions. They also manually inspected changes made in the tracked clone groups.

We have tracked the clone groups detected by SimScan including the five clone groups above and reconstructed change histories for them by our method. We used SimScan with the same settings as that of Duala-Ekoko and Robillard's experiment, namely volume=medium, similarity=fairly similar, and speed=fast. Our tracking results were consistent with their results except for a clone region that they could not track. Our system was able to track the clone region up to the most recent version. All reported changes collected by their manual inspection were automatically reconstructed by our change history construction method briefly described in Sect. 3.4.

We also pairwise compared reconstructed change histories of code fragments in tracked clone groups. We set the similarity threshold to 0.5. For lack of space, we only report the results of Ant. 537 clone pairs out of 1,078 initial clone pairs detected by SimScan were inconsistently modified without disappearing before the latest version, while 340 pairs disappeared before the latest version. Our system also detected 1,247 additional clone pairs after the initial version. Among them, 272 pairs were inconsistently modified (excluding 369 disappeared clone pairs). Note that detected inconsistent changes do not immediately account for bugs. There is a possibility that clones are intentionally modified differently [12]. It took our system 60 and 70 minutes to complete the whole analysis of jEdit and Ant, respectively.

In our system, cloning activities can be visualized using continua. Figures 7 and 8 show file and code (MiscUtilities.java) continua for jEdit. Each horizontal line represents a file (node) continuum and each polygon a group of file (node) continua that begin at the same version. In each polygon, continua are sorted by terminating versions and colors represent the percentage of continua generated by cloning activities in the corresponding group. We can see that a couple of versions introduced numerous clones.

\subsection{Linux Device Drivers}

A case study is reported, in which investigation of inconsistent changes detected by our system has led to a certain contribution to an open source community. We collected and analyzed 32 versions of Linux 2.6 kernel source code from 2.6.0 


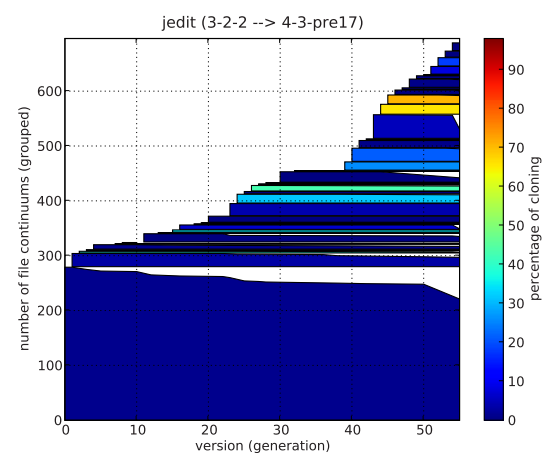

Fig. 7. File Continua of $\mathrm{jEdit}$

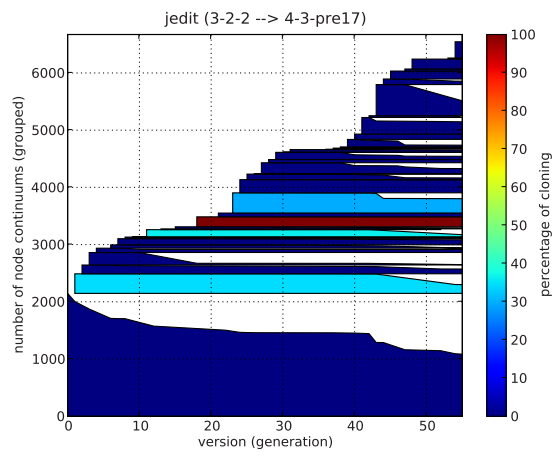

Fig. 8. Node Continua of jEdit (MiscUtilities.java)

to 2.6.31. We first detected and tracked the paralogues in the entire kernel source code. Then we constructed fine-grained change histories for the paralogues in the drivers subsystem and inspected the histories to detect inconsistent changes. The drivers subsystem itself is a large code base as it occupies more than $70 \%$ of modern operating systems in volume and accounts for the vast majority of bugs as reported by Chou and others [13.

In order to detect initial clones in the initial version 2.6.0, we used CCFinder with the default setting. After CCFinder had detected 2,851 clone pairs, we set the similarity threshold between change histories to 0.9999 and ran the system. It took three days to detect additional clone pairs and to track all paralogues. It then took two more days to construct change histories for paralogues in the drivers subsystem. The system detected 814 additional clone pairs (cloning activities) after version 2.6.0, and 1,441 and 385 inconsistently modified pairs out of the initial and the additional clone pairs, respectively. After automated analysis is completed, we manually inspected inconsistent changes with similarity scores more than or equal to 0.9 . It should be noted that overlooking changes such as argument deleted and parameter type changed leads to compiler errors, and hence immediate regression faults. Overlooking protocol changes such as function call inserted, however, often causes latent errors difficult to detect. Thus we focused on insertion of statements.

We were able to find approximately 10 inconsistent changes involving insertions of function calls to lock_kernel that remained in the latest version 2.6.31. An inconsistent change involving lock_kernel detected by the system is shown in Fig. 9. where a clone pair in synclinkmp.c and synclink_cs.c was inconsistently modified. Their change type sequences were mostly the same, which means that they almost consistently co-evolved, but differ in only one change. In this experiment, we observed a number of clone pairs that evolved almost consistently. Among them, a pair of change histories that shares 178 change types in common with a similarity score of 0.98 was discovered.

By further inspection, we found that the inconsistency relates to similar inconsistencies observed in Linux serial drivers violates a development policy 


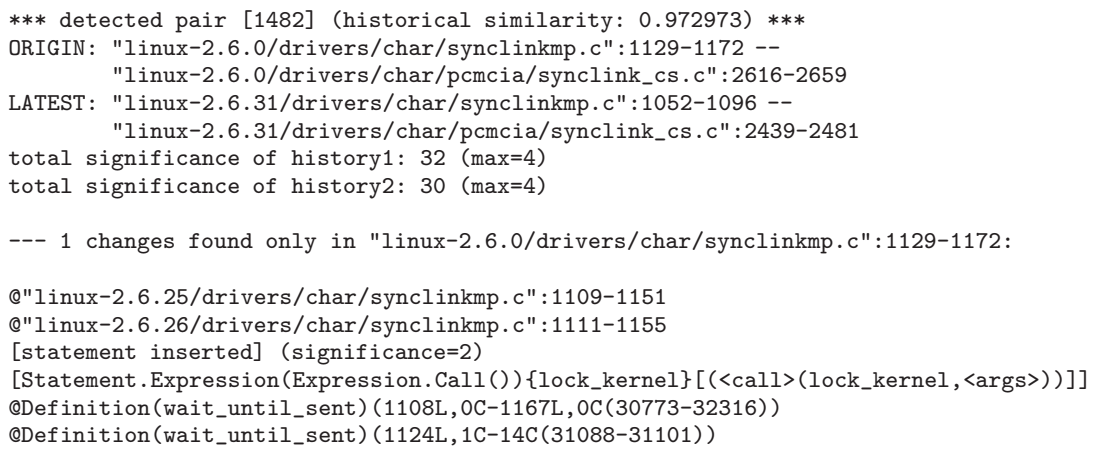

Fig. 9. An Example of Inconsistent Change

concerning kernel locking known as BKL (big kernel lock) pushdown. The BKL was introduced to make the kernel work on multi-processor systems. The role of the BKL, however, has diminished over years since fine-grained locking has been implemented throughout the kernel for better throughput. Although some attempts to entirely remove the BKL have been made, progress in that direction has been slow in recent years.

It was not long before the BKL accounted for a performance regression. At last, some of the developers decided to go a step further in versions 2.6.26 and 2.6.27. They began with serial drivers. In order to remove upper-level acquisition of the BKL in the control flows, they attempted to push the acquisition down to the device specific code level, where they expected BKL removal to be achieved. Indeed, numerous lock_kernel calls were (almost blindly for safety) inserted into serial driver code including synclinkmp.c at version 2.6.26, and then the upperlevel call (in fs/char_dev.c) was removed at version 2.6.27. However, during the pushdown, synclink_cs.c was left unchanged, which led to an inconsistent change detected by our system.

Although the inadvertency itself does not cause errors, we could promote the BKL pushdown policy. In response to our report on the inconsistent change, the author of synclinkmp.c pointed out that the lock_kernel calls in the driver can be safely removed. We submitted a patch removing the BKL related calls from synclinkmp.c and its variations. It was acknowledged by the author of the driver.

\section{Related Work}

There is a large body of studies on code clones and related detection tools 44. Code clone detection and source code differencing are two complementary techniques for analyzing relationship among software products. Although code clone detection tools may well be able to discover homologous code, they may not be suitable for precisely tracking life cycles of such code in the long evolution of the software as it requires consistently identifying changed and unchanged parts. It 
usually takes awkward steps of taking difference by way of clone detection. Since code clone detection methods can analyze clones across different and unrelated software products, it would be an interesting topic to combine these two methods. One idea is to use clone detection tools first to reduce the size and the scope of the problem, and then to apply tree differencing tools for detailed analysis.

Kim and others [7] proposed a method for inferring high-level description of structural changes based on the first-order logic in order to determine method level matches between versions of Java programs. While their method is specialized for Java programs, their idea of aggregating low-level description of changes seems applicable to our system. Kim and Notkin apply clone detection techniques to understand evolution of code clones [5] in Java software. They rely on location overlapping relationship to track code snippets across multiple versions of a program. While their analysis is simple and fast, it may not be able to extract how such code snippets changes over time from the source code. Duala-Ekoko and Robillard 6] proposes a code tracking method tailored for Java. Although the method is driven by heuristics and suitable for interactive use, the lack of precision in syntactic analysis may limit the ability of the tool. Godfrey and Zou [14] developed a set of techniques for detecting merging/splitting of functions and files in software systems. They presented a set of merge/split patterns and employed call relationships to aid in detecting their occurrence, which are also useful for our analysis. Aversano and others [15] proposed a method of investigating how clones are maintained over time. In order to derive evolution patterns of clones, they rely on fast but coarse line-by-line differencing to track clones.

In Sect. 3, we implicitly assumed that the genealogies of target software systems are given. However, without any development history or any explicit record of tagging or version copying operations, it may be difficult to determine the origin of branching, notably the version from which a development branch is duplicated. In such situations, we can reconstruct the genealogies by utilizing tree differencing and tools for phylogeny [9].

\section{Conclusion}

We have proposed an automated method for analyzing code homology in genealogy of evolving software based on fine-grained tree differencing. Homologues can be introduced through various activities: branching/forking in software projects (orthologues), code exchange between neighboring branches such as code import/ merging and common bug-fix patches (xenologues), and code duplication within branches (paralogues or code clones). As the development proceeds, homologues can incur additional maintenance efforts. We have developed a method for detecting and tracking such distinctive pieces of code by exploiting fine-grained tree differencing. Detecting and tracking homologues along evolution branches enable us to reconstruct and to compare change histories of homologues, which leads us to detect inconsistent changes. Results of experiments conducted on several large-scale software including BIND9 DNS servers, a couple of Java software jEdit and Ant, and Linux device drivers have been reported to show the capability 
of the method. Having scalable and precise tree differencing engines helped us to analyze a large-scale software project such as the Linux kernel consisting of several millions of SLOC.

Future work includes the following:

1. to improve processing speed by further exploiting parallelism and by eliminating redundant computation,

2. to build a database for efficiently storing and retrieving various (intermediate) results computed by the system, together with comprehensive graphical user interface, and

3. to apply our analysis to:

(a) change pattern mining and future modification prediction,

(b) language-aware merging, and

(c) the concrete problem of generating generic patches [16 that cover wide range of Linux device drivers.

\section{References}

1. Fitch, W.: Homology a personal view on some of the problems. Trends in Genetics 16(5), 227-231 (2000)

2. Fluri, B., Gall, H.C.: Classifying change types for qualifying change couplings. In: ICPC 2006: Proceedings of the 14th IEEE International Conference on Program Comprehension, pp. 35-45. IEEE Computer Society, Los Alamitos (2006)

3. Kim, M., Notkin, D.: Program element matching for multi-version program analyses. In: MSR 2006: Proceedings of the 2006 international workshop on Mining software repositories, pp. 58-64. ACM, New York (2006)

4. Roy, C.K., Cordy, J.R., Koschke, R.: Comparison and evaluation of code clone detection techniques and tools: A qualitative approach. Science of Computer Programming 74(7), 470-495 (2009)

5. Kim, M., Sazawal, V., Notkin, D., Murphy, G.: An empirical study of code clone genealogies. In: ESEC/FSE-13: Proceedings of the 10th European software engineering conference held jointly with 13th ACM SIGSOFT international symposium on Foundations of software engineering, pp. 187-196. ACM, New York (2005)

6. Duala-Ekoko, E., Robillard, M.P.: Tracking code clones in evolving software. In: ICSE 2007: Proceedings of the 29th International Conference on Software Engineering, pp. 158-167. IEEE Computer Society, Los Alamitos (2007)

7. Kim, M., Notkin, D., Grossman, D.: Automatic inference of structural changes for matching across program versions. In: ICSE 2007: Proceedings of the 29th international conference on Software Engineering, pp. 333-343. IEEE Computer Society, Los Alamitos (2007)

8. Reiss, S.P.: Tracking source locations. In: ICSE 2008: Proceedings of the 30th international conference on Software engineering, pp. 11-20. ACM, New York (2008)

9. Hashimoto, M., Mori, A.: Diff/TS: A tool for fine-grained structural change analysis. In: WCRE 2008: Proceedings of the 15th Working Conference on Reverse Engineering, pp. 279-288. IEEE Computer Society, Los Alamitos (2008)

10. Kamiya, T., Kusumoto, S., Inoue, K.: Ccfinder: A multilinguistic token-based code clone detection system for large scale source code. IEEE Transactions on Software Engineering 28(7), 654-670 (2002) 
11. Myers, E.W.: An O(ND) difference algorithm and its variations. Algorithmica 1(2), 251-266 (1986)

12. Kapser, C., Godfrey, M.W.: "Cloning considered harmful" considered harmful. In: WCRE 2006: Proceedings of the 13th Working Conference on Reverse Engineering, pp. 19-28. IEEE Computer Society, Los Alamitos (2006)

13. Chou, A., Yang, J., Chelf, B., Hallem, S., Engler, D.: An empirical study of operating systems errors. In: SOSP 2001: Proceedings of the eighteenth ACM symposium on Operating systems principles, pp. 73-88. ACM, New York (2001)

14. Godfrey, M.W., Zou, L.: Using origin analysis to detect merging and splitting of source code entities. IEEE Transactions on Software Engineering 31(2), 166-181 (2005)

15. Aversano, L., Cerulo, L., Di Penta, M.: How clones are maintained: An empirical study. In: CSMR 2007: Proceedings of the 11th European Conference on Software Maintenance and Reengineering, pp. 81-90. IEEE Computer Society, Los Alamitos (2007)

16. Padioleau, Y., Lawall, J.L., Muller, G.: Understanding collateral evolution in linux device drivers. In: EuroSys 2006: Proceedings of the 1st ACM SIGOPS/EuroSys European Conference on Computer Systems 2006, pp. 59-71. ACM, New York (2006) 